\title{
Turbo Field Echo MRI
}

National Cancer Institute

\section{Source}

National Cancer Institute. Turbo Field Echo MRI. NCI Thesaurus. Code C118461.

A magnetic resonance imaging modality that uses a gradient echo pulse sequence with data acquisition after an initial 180 deg ree preparation pulse for contrast enhancement. TFE provides contrast at shorter echo times and acquisition times, thus reducing related image artifacts. 